\title{
Functional Shift During Orthodontic Correction of Class II Division 2 Malocclusion in an Adult- A Rare Case Report
}

\author{
Sanjay Prasad Gupta
}

Assistant Professor, Orthodontics and Dentofacial Orthopedics unit, Department of Dentistry, Tribhuvan University Teaching Hospital, Institute of Medicine, Kathmandu, Nepal.

sanjayagupta2000@gmail.com

*Corresponding Author: Sanjay Prasad Gupta, Assistant Professor, Orthodontics and Dentofacial Orthopedics unit, Department of Dentistry, Tribhuvan University Teaching Hospital, Institute of Medicine, Kathmandu, Nepal.

\section{Abstract}

The prevalence of Angle Class II Division 2 malocclusion is relatively low in comparison with other malocclusions. This case report describes the management of an adult female patient with a class II division2 malocclusion with retroclined upper central incisors, proclined upper lateral incisors, crowding, deep bite and retrussive lips through comprehensive orthodontic treatment. This case was treated on non-extraction basis using 0.022 slot MBT preadjusted edgewise appliance. Treatment was completed in 9.5 months with good occlusion and facial esthetics. Anterior shift occurred in this case as the incisal interference were removed and turned Class I from Class II malocclusion.

Keywords: Functional shift, Class II malocclusion, Non extraction orthodontic treatment

\section{INTRODUCTION}

Angle ${ }^{1}$ proposed a classification system based on the relationship of the mandibular first molars to the maxillary first molars. He characterized the Class II malocclusions as having a distal relationship of the mandibular teeth relative to the maxillary teeth of more than one-half the width of the cusp.

Angle ${ }^{1}$ characterized two types of Class II malocclusions based on the inclination of the maxillary central incisors. Class II Division 1 malocclusions are described as having labially inclined maxillary incisors, an increased overjet with or without a relatively narrow maxillary arch. The Class II Division 2 malocclusions are described as having excessive lingual inclination of the maxillary central incisors overlapped on the labial by the maxillary lateral incisors. In some cases, both the central and the lateral incisors are lingually inclined and the canines overlap the lateral incisors on the labial. ${ }^{1}$

This type of malocclusion is usually transformed into a Class II Division 1 malocclusion by proclination of the maxillary incisors and then treated as a Division 1 malocclusion. ${ }^{3}$ However, because of some specific morphologic characteristics, including retroclination of the maxillary incisors, deep bite with a tendency to a brachycephalic facial pattern, and poor softtissue profile, a nonextraction approach to treat Class II Division 2 malocclusions is recommended. Practically, the combination of orthodontic treatment and surgical intervention might be required to obtain ideal esthetic and functional results for an adult with this combination of facial and skeletal problems.

The Class II Division 2 malocclusion is often accompanied by a deep overbite and minimal overjet. In cases with extreme overbite, the incisal edges of the lower incisors may contact the soft tissues of the palate. ${ }^{4,5}$ In a few Class II Division 2 cases, the mandibular labial gingival tissues may be also traumatized by the lingually inclined maxillary incisors, particularly in the absence of an overjet.

An exaggerated curve of Spee may be present in the mandibular arch with extrusion of the mandibular incisors. ${ }^{6}$ 
Functional Shift During Orthodontic Correction of Class II Division 2 Malocclusion in an Adult- A Rare Case report

\section{INCIDENCE}

Ast and coworkers ${ }^{7}$ found that the incidence of Class II Division 2 to be $3.4 \%$ and for Class II Division 2 subdivision to be $1.6 \%$. When compared with other malocclusions Class II Division 2 occurs less frequently than either Class I or Class II Division 1, but slightly more frequently than Class III. ${ }^{7}$

Wallis ${ }^{8}$ compared Class II Division 2, Class I and Class II Division 1 individuals and found that the posterior cranial base was larger in Division 2 cases. He also noted that the mandibular form in a "typical" Division 2 case has relatively more acute gonial and mandibular plane angles, shorter lower anterior face height, and excessive overbite.

Peck and Peck (1998) ${ }^{9}$ considered Class II division 2 as heritable having strong familial occurrence. While Ruf \& Pancherz (1999) ${ }^{10}$ reported monozygotic twins showing discordance. Based on this report they said heredity is not the sole aetiological factor as normally one would expect similar occlusion in monozygotic twins.

\section{Path of Closure}

It has also been suggested that as the mandible is brought from the postural resting position to habitual occlusion in some Class II Division 2 cases, the path of closure is influenced by the lingually inclined maxillary incisors together with the infraocclusion of the posterior teeth. ${ }^{11}$

The combination of these two factors results in an abnormal path of mandibular closure as well as overclosure. More specifically, the mandible is forced into a retruded position by the anterior teeth and the condyles are displaced posteriorly and superiorly in the articular fossa. ${ }^{11}$

The presence of such a "posterior functional shift," in some cases, may favorably influence the prognosis for the correction of the Class II relationship. Swan ${ }^{12}$ estimated that one-third of the cases exhibited a functional component that allowed for a partial correction of the malocclusion following the labial repositioning of the maxillary incisors. The creation of the overjet during treatment in such cases allowed the mandible to move forward to a normal centric relation position.
It is important to emphasize that clinicians should be aware of this possibility, but they should not assume that it is a consistent finding in Class II Division 2 cases.

This malocclusion is also associated with an abnormal pattern of mandibular posture and closure that makes the diagnosis and treatment most intriguing. ${ }^{13}$

Treatment of an adult Class II patient requires careful diagnosis and a treatment plan involving esthetic, occlusal, and functional considerations. ${ }^{14}$ The treatment objectives must include the chief complaint of the patient, and the mechanics plan should be individualized based on the specific treatment goals.

The excessive lingual inclination of the maxillary incisors might have resulted in a functional mandibular retrusion. This could be determined by "freeing" the mandible either by tipping the maxillary central incisors labially or by placing a bite plate to disarticulate the anterior teeth allowing the mandible to assume a position dictated by the musculature. When a shift is present, the anterior movement of the mandible will be advantageous in the treatment of the malocclusion. Furthermore, the labial movement of the maxillary incisors will facilitate the uncrowding of the mandibular incisors by allowing the tongue and lip musculature to establish the position of the lower incisors without the confining influence of the lingually tipped maxillary incisors.

Several treatment options are available for correction of class II div 2 cases depending on the growth and severity of the skeletal jaw base discrepancy in antero-posterior and vertical direction. In Class II patients with mild-to-moderate skeletal discrepancies, ${ }^{15}$ orthodontic camouflage may well be the treatment of choice. It involves intrusion and proclination of the upper incisors during the initial phase of treatment, thereby unlocking the malocclusion that in turn permits a modification in the path of closure of mandible and ${ }^{16,17}$ aids in the correction of Class II molar relationship.

This case report illustrates orthodontic treatment by non extraction in adult female having skeletal Class II along with dental Class II Division 2 malocclusion. 
Functional Shift During Orthodontic Correction of Class II Division 2 Malocclusion in an Adult- A Rare Case report

\section{CASE Report}

\section{Diagnosis and Etiology}

A 25-year-old woman was referred for orthodontic consultation (Fig.1).Her chief complaints was irregular teeth in upper front region of jaw. She had no relevant family history, no significant prenatal, postnatal and medical history and no history of parafunctional habits. On functional examination, there was no signs and symptoms of temporomandibular joint dysfunction.
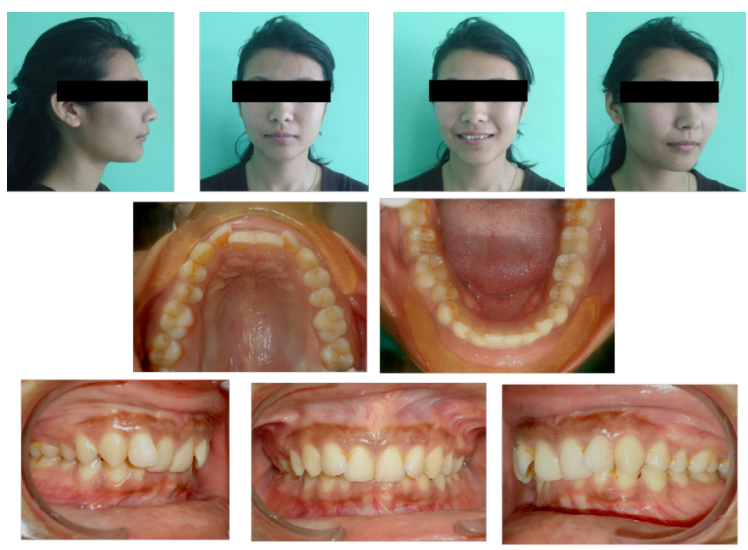

Figure 1. Pretreatment Intraoral and Extraoral Photographs

On clinical examination, she had a convex profile with a symmetric face and lip competence at rest.

Intraorally, there was a Class II molar and canine relationship bilaterally. The overbite was excessive, and the lingually inclined maxillary incisors were overerupted and impinging on the attached labial gingiva of the mandibular incisors with labially tipped maxillarylateral incisors creating an unattractive smile. The overerupted mandibular incisors also impinged on the palatal gingiva, lingual to the maxillary incisors having overjet of $1.5 \mathrm{~mm}$ and overbite of $9 \mathrm{~mm}$.

The both maxillary and mandibular arch were U-shaped and had mild crowding in the incisor segment with an excessive curve of spee bilaterally.

The cephalometeric analysis showed a skeletal Class II antero posterior discrepancy (Fig.2) with an ANB angle of $8^{\circ}$ and a horizontal growth pattern, as shown by an FMA of $23^{\circ}$ and SN-GoGN of $28.5^{\circ}$. Severely retroclined maxillary incisors caused an obtuse nasolabial angle of $95^{\circ}$.

The panoramic radiograph showed the presence of all third molars. The overall alveolar bone level was within normal limits (Fig.3).
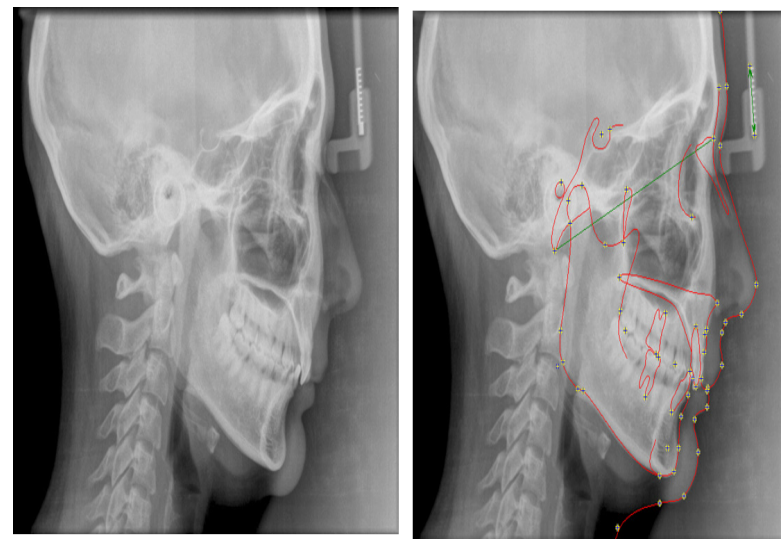

Figure 2. Pretreatment lateral cephalograms

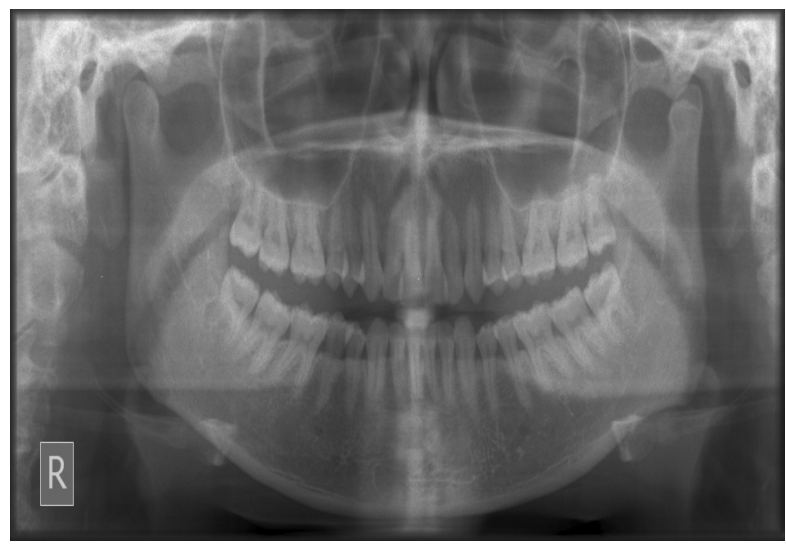

Figure 3. Pretreatment orthopantamogram

\section{Treatment Objectives}

The treatment objectives were to correct the deep overbite, establish class I canine relation, improve the smile arc and soft tissue esthetics.

\section{Treatment Alternatives}

Treatment options for correction of skeletal Class II Div 2 malocclusion in adults include orthodontic camouflage or orthognathic surgery. However, this patient was treated with orthodontic camouflage because the severity of the sagittal and vertical jaw base discrepancy did not warrant surgery. Moreover, long term studies of Class II malocclusion comparing camouflage and surgery showed similar reports of overall satisfaction with both the treatment modalities, with the camouflage group having fewer functional and temporomandibular problems ${ }^{18}$.

\section{Treatment Progress}

The maxillary teeth were bonded with fully programmed Preadjusted 0.022 MBT prescription 
Functional Shift During Orthodontic Correction of Class II Division 2 Malocclusion in an Adult- A Rare Case report

brackets. Brackets were bonded in the lower arch after sufficient overjet was achieved and the arches were aligned using the following sequence of archwires; 0.012 Niti, 0.014 Niti and 0.016 Niti. Surprisingly the slight mandibular shift occurred as the upper incisor interferences were removed that brings the class II molar relation to class I molar relation. Later, 0.018ss wire followed by $0.019 \times 0.025$ stainless steel arch wire was placed to level and improve the torque of the upper incisors (Fig.4). Class II and Class III elastics was given for midline correction and proper interdigitation during the settling phase. Finishing and detailing was done and the appliance was debonded. The total treatment time was 9.5 months.
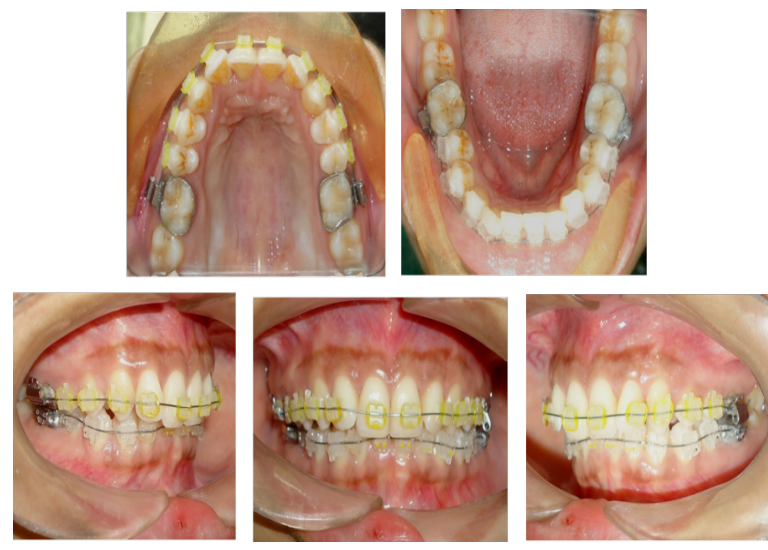

Figure 4. Mid treatment photographs

\section{Treatment Results}

The post treatment facial photographs showed a remarkable improvement in patient profile and facial esthetics. Facial balance and smile esthetics were improved. Lip support improved for both upper and lower lip (Fig. 5).

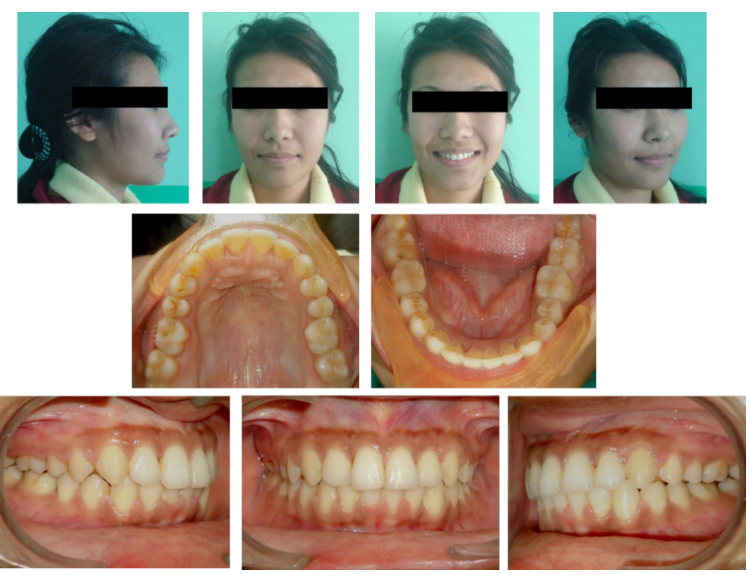

Figure 5. Post treatment extraoral and intraoral Photographs
Intraorally, an optimal overbite and overjet relationship was established. A well-interdigitated buccal occlusion with Class I canine and molar relationships was created. Overjet and overbite relationship was improved to 2.5 $\mathrm{mm}$ and $3 \mathrm{~mm}$ respectively. There was canine guidance in lateral excursions with proper anterior guidance without balancing side interferences.

The posttreatment cephalometric radiograph (Fig.6) and superimposed tracings (Fig.7) showed significant changes in the dental and skeletal measurements after treatment.
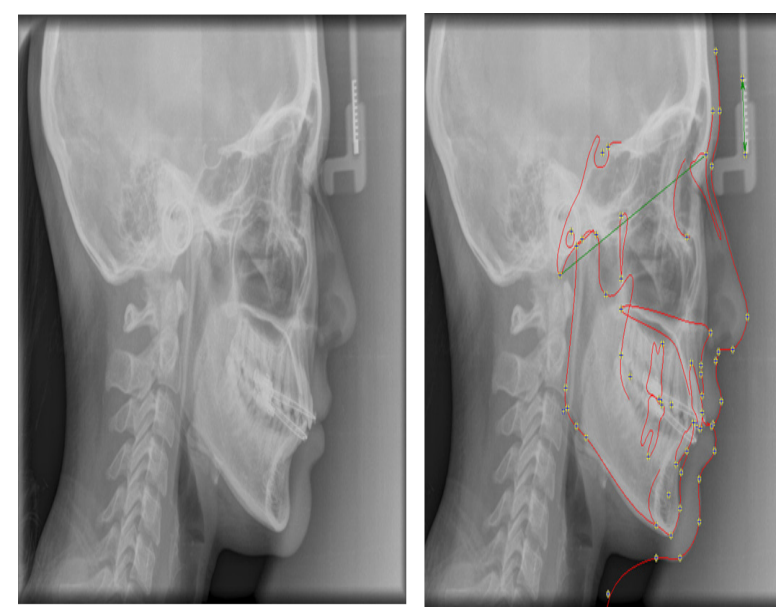

Figure 6. Post treatment cephalograms
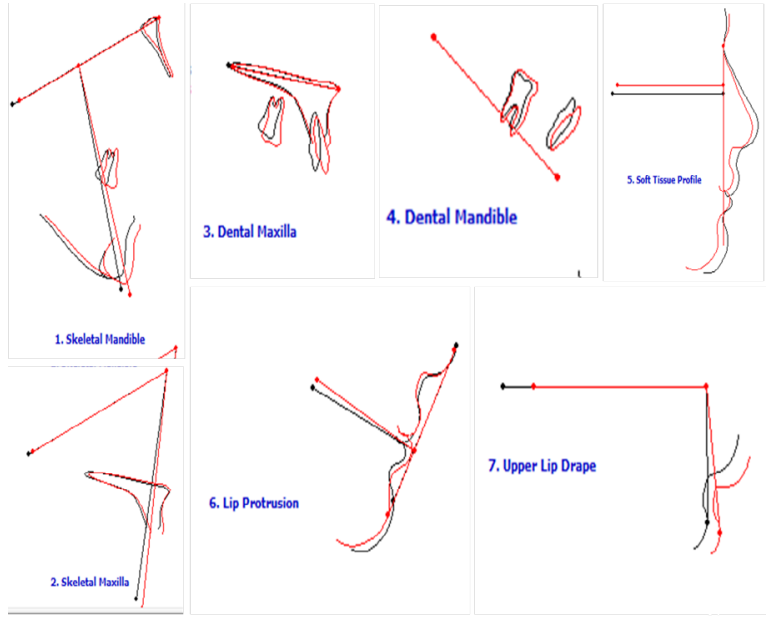

Figure 7. Superimposed tracing

Significant intrusion and proper proclination of the maxillary incisors were achieved. The lip profile improved significantly (Fig 5).

The pretreatment and post treatment cephalometric parameters is compared in Table no. 1 . 
Functional Shift During Orthodontic Correction of Class II Division 2 Malocclusion in an Adult- A Rare Case report

Table 1. Comparative cephalometric parameters

\begin{tabular}{|c|c|c|c|}
\hline Cephalometric parameters & Clinical norms & Pre-treatment values & Post-treatment values \\
\hline SNA & $82 \pm 2^{\circ}$ & $88^{0}$ & $88^{0}$ \\
\hline SNB & $80 \pm 2^{\circ}$ & $80^{\circ}$ & $82^{\circ}$ \\
\hline ANB & $2 \pm 2$ o & $8^{\circ}$ & $6^{0}$ \\
\hline Wits & $0-(-) 1$ & $2 \mathrm{~mm}$ & $0 \mathrm{~mm}$ \\
\hline FMA & $25 \pm 2^{\circ}$ & $23^{\circ}$ & $27^{\circ}$ \\
\hline SN-GoGn & $32 \pm 2^{\circ}$ & $28.5^{0}$ & $31^{\circ}$ \\
\hline Max.I-NA & $22 \pm 2^{\circ}$ & $13^{\circ}$ & $20^{\circ}$ \\
\hline Man.I-NB & $25 \pm 2^{\circ}$ & $26.6^{\circ}$ & $29^{\circ}$ \\
\hline LI-A-Pog & $2.7 \pm 1.7 \mathrm{~mm}$ & $-4 \mathrm{~mm}$ & $0 \mathrm{~mm}$ \\
\hline IMPA & $90 \pm 2^{\circ}$ & $96^{\circ}$ & $97^{\circ}$ \\
\hline Interincisal angle & $134^{\circ}$ & $142^{\circ}$ & $125^{\circ}$ \\
\hline
\end{tabular}

The posttreatment panoramic radiograph showed good root parallelism. (Fig.8)

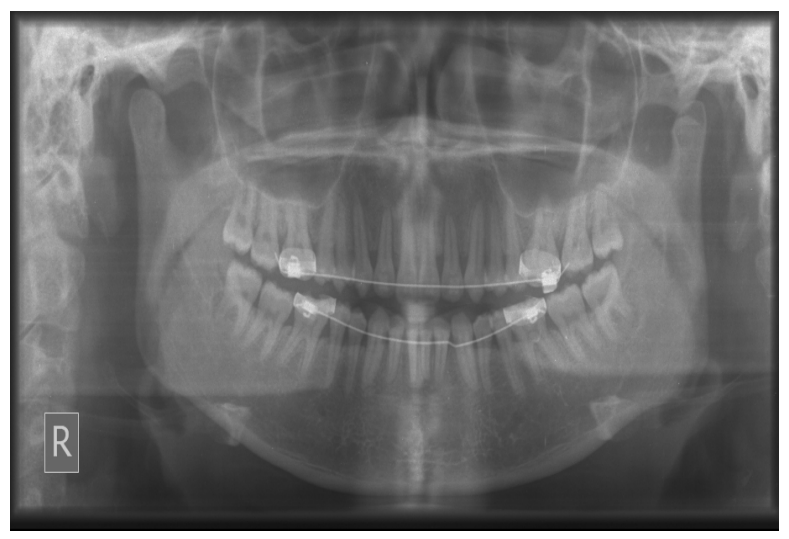

Figure 8. Post treatment orthopantamograms

\section{DISCUSSION}

The morphological characteristics of a Class II Division 2 malocclusion include deep overbite and retroclination of the maxillary incisors. This leads to a common belief that the mandible, during closure from the rest position into the intercuspal position, can displace posteriorly and contribute to the development of temporomandibulardisordersymptoms. 19,20

It is assumed that, after intrusion and proclination of the maxillary incisors, the mandible will spontaneously move forward and accordingly simplify the correction of the Class II skeletal and anteroposterior dental discrepancies, especially in nongrowingpatients. ${ }^{21}$ However, this concept is controversial, and some studies have shown that, in a patient with a Class II Division 2 malocclusion, the mandible is not displaced backward. ${ }^{19,22}$
Most clinicians agree that when possible, the treatment of Class II Division 2 malocclusions with a low mandibular plane angle and deep overbite are best managed with a nonextraction approach to avoid retraction of the incisors and protraction of the molars; both of these movements tend to further deepen the overbite. ${ }^{23}$ On the other hand, with a nonextraction approach, the labial movement of the lower incisors during leveling as well as the distal movement and extrusion of the maxillary molars with various mechanics would help in the correction of the deep overbite.

Another critical parameter to consider in the extraction decision is the patient's profile. Many individuals with Class II Division 2 malocclusions have relatively retrusive lips as well as prominent chins and noses. Extraction of premolars followed by incisor and lip retraction will further retrude the lips. Such an outcome would worsen the profile and will result in an unacceptable "edentulous look."

The decision of whether to extract or not can only be determined through the proper diagnosis of each case. Before considering the extraction of premolars, the clinician needs to evaluate several factors including the prominence of the nose and chin, the presence of a functional mandibular retrusion, the patient growth potential and headgear cooperation, the extent of the tooth size-arch length discrepancy, and the periodontal condition of the lower anterior teeth. As a rule, in borderline crowded Class II Division 2 cases, it would be prudent to start the treatment with a nonextraction approach. 
Functional Shift During Orthodontic Correction of Class II Division 2 Malocclusion in an Adult- A Rare Case report

Strang ${ }^{6}$ believes that with good vertical growth during treatment, the overbite can be successfully corrected by intruding the anterior teeth. He suggested that in these very deep overbite cases, the extrusion of the posterior teeth in the absence of vertical growth will result in a muscular imbalance that will cause a relapse of the corrected overbite. ${ }^{24}$

Schudy, ${ }^{25}$ on the other hand, advocates extrusion of the posterior teeth particularly in patients with a decreased lower face height, a flat mandibular plane angle, and a prominent chin.

\section{CONCLUSION}

Treatment of Class II, division 2 malocclusion in adults is always challenging. Functional mandibular retrusion may alleviate the need of extraction \& improve esthetics. Extraction decision can only be determined through proper diagnosis.

\section{REFERENCES}

[1] Angle EH: Treatment of Malocclusion of the Teeth, $7^{\text {th }}$ ed. Philadelphia, SS White, 1907.

[2] Graber TM: Overbite-the dentist's challenge. J Am Dent Assoc 79:1135-1145, 1969.

[3] Tuncay OC, Tulloch JFC. Apparatus criticus: methods used to evaluate growth modification in Class II malocclusion. Am J Orthod Dentofacial Orthop 1992;102:531-6.

[4] Sassouni J: A classification of skeletal facial types. Am J Orthod 55:109-123, 1969.

[5] Sassouni J: The Class II syndrome: differential diagnosis and treatment. Angle Orthod 40:334-341, 1970.

[6] Strang RHW: Class II Division 2 malocclusion. Angle Orthod 28:210-214, 1958.

[7] Ast DB, Carlos JP, Cons DC: Prevalence and characteristics of malocclusion among senior high school students in up-state New York. Am J Orthod 51:437-445, 1965.

[8] Wallis SF: Integration of certain variants of the facial skeleton in Class II Division 2 malocclusion. Angle Orthod. 33:1, 60-67, 1963.

[9] Peck S, Peck L, Kataja M. Class II Division 2 malocclusion: a heritable pattern of small teeth in well-developed jaws. Angle Orthod 1998;68:9-20.
[10] While Ruf \& Pancherz. Class II Division 2 malocclusion: genetics or environment? A case report of monozygotic twins. Angle Orthod 1999 Aug;69(4):321-4.

[11] Graber TM: Overbite-the dentist's challenge. J Am Dent Assoc 79:1135-1145, 1969.

[12] Swann GC: The diagnosis and interpretation of Class II, Division 2 malocclusion. Am J Orthod 40:325-340, 1954.

[13] Leity P.Erickson, W.Stuart Hunter Class II div 2 treatment and mandibular growth. Angle Orthod 1985,vol 55 No 3 215-224.

[14] Nanda R. Treatment of Class II, Division 2 Malocclusion in Adults: Biomechanical Considerations. J Cli Orthod 2003 Nov;37(11):599-606; quiz 625.

[15] Flavio Uribe, Ravindra Nanda.Treatment of Class II, Division 2 Malocclusion in Adults: Biomechanical Considerations. JCO 2003 vol XXXVII No11599-606.

[16] Leity P.Erickson, W.Stuar t Hunter Class II div 2 treatment and mandibular growth. Angle Orthod 1985,vol 55 No 3 215-224.

[17] John F . Cleall and Ellen A. Begole Diagnosis and treatment of Class II Div 2 malocclusions Angle Orthod 1982;vol 52 No; 38-60.

[18] Colin A. Mihalik, William R. Proffit and Ceib Phillips. Long-term follow-up of Class II adults treated with orthodontic camouflage: A comparison with orthognathic surgery outcomes. AJODO 2003;123;266-78

[19] Demisch A, Ingervall B, Thüer U. Mandibular displacement in Angle Class II, Division 2 malocclusion. Am J Orthodod Dentofacial Orthop 1992;102:509-18.

[20] Thompson JR. Abnormal function of the temporomandibular joints and related musculature. Orthodontic implications. Part II. Angle Orthod 1986;56:181-95.

[21] Owen AH. Orthodontic/orthopedic treatment of craniomandibular pain dysfunction. Part 2: posterior condylar displacement. J Craniomand Pract 1984:2:334-49. 
Functional Shift During Orthodontic Correction of Class II Division 2 Malocclusion in an Adult- A Rare Case report

[22] Cleall JF, BeGole EA. Diagnosis and treatment of Class II, Division 2 malocclusion. Angle Orthod 1982;52:38-60.

[23] A new look at the treatment of Class II Division 2 malocclusions. Joseph Yousefian, A Douglas Trimble, b and Gary Folkman c (Am J Orthod Dentofacial Orthop 2006;130:771-8)
[24] Samir E. Bishara: Class II Malocclusions: Diagnostic and Clinical Considerations With and Without Treatment, Semin Orthod 2006;12:11-24.

[25] Schudy FS: The control of vertical overbite in clinical orthodontics. Angle Orthod 38:19-39, 1968

Citation: Sanjay Prasad Gupta. Functional Shift During Orthodontic Correction of Class II Division 2 Malocclusion in an Adult- A Rare Case report. Archives of Dentistry and Oral Health. 2018; 1(1): 22-28.

Copyright: (C) 2018 Sanjay Prasad Gupta. This is an open access article distributed under the Creative Commons Attribution License, which permits unrestricted use, distribution, and reproduction in any medium, provided the original work is properly cited. 